\title{
AN INSTITUTIONAL STUDY TO EVALUATE THE EFFICACY AND TOXICITY OF REIRRADIATION USING CONVENTIONAL RADIOTHERAPY FOR RECURRENT MALIGNANT DISEASE
}

\author{
Sudhir Katariya ${ }^{1}$, Manish Verma $^{2}$, Arpit Jain $^{3}$, Vinay Shivhare ${ }^{4}$, Allwin George ${ }^{5}$, Minaal Iyer ${ }^{6}$, Ashish Malviya ${ }^{7}$, Abhishek Pratap Singh ${ }^{8}$ \\ ${ }_{1}^{1}$ Specialist Medical Officer, Department of Radiation Oncology, Government Cancer Hospital, MGMMC, Indore, Madhya Pradesh, India. \\ ${ }^{2}$ Assistant Professor, Department of Radiation Oncology, Government Cancer Hospital, MGMMC, Indore, Madhya Pradesh, India. \\ $33^{\text {rd }}$ Year Postgraduate Student, Department of Radiation Oncology, Government Cancer Hospital, MGMMC, Indore, Madhya Pradesh, \\ India. \\ ${ }^{4}$ Senior Resident, Department of Radiation Oncology, Government Cancer Hospital, MGMMC, Indore, Madhya Pradesh, India. \\ ${ }_{5}^{5}$ Senior Resident, Department of Radiation Oncology, Government Cancer Hospital, MGMMC, Indore, Madhya Pradesh, India. \\ ${ }^{6}$ Senior Resident, Department of Radiation Oncology, Government Cancer Hospital, MGMMC, Indore, Madhya Pradesh, India.

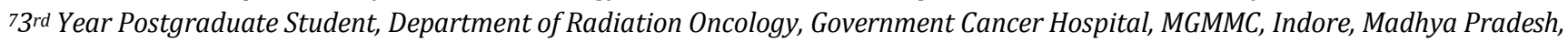 \\ India. \\ ${ }^{8} 2^{\text {nd }}$ Year Postgraduate Student, Department of Radiation Oncology, Government Cancer Hospital, MGMMC, Indore, Madhya Pradesh, \\ India.
}

\section{ABSTRACT}

\section{BACKGROUND}

Locoregional failure is the most frequent pattern of failure in various cancer patients despite intensified treatment and it leads to death in most of the patients. Traditionally, surgery as salvage therapy for locoregional recurrence provides the greatest probability of cure; unfortunately, the vast majority of locoregional recurrent cases are unresectable. Although chemotherapy has been the standard of care for patients who are not candidates for surgery, the response rates are limited. Reirradiation is a potentially curative or best palliative treatment option for some patients with unresectable disease. This study reported our experience of reirradiation alone or concurrent chemoradiotherapy in recurrent malignant condition.

\section{MATERIALS AND METHODS}

We reviewed 26 consecutive patients with a diagnosis of locoregional recurrences (LRR) of Head and Neck, Cervix, Urinary Bladder, CNS carcinoma treated with a second course of radiotherapy. The main outcome measures were local control, overall survival, and complications.

\section{RESULTS}

Efficacy and toxicity were scored by looking at the reduction in tumour size, pain and bleeding. Reduction in tumour size and pain reduction after reirradiation were seen in $100 \%$ and $95 \%$ of the patients respectively. In $95 \%$ of the cervical carcinoma patients there was a reduction in bleeding. The 1-year local control rate for the whole group was $64 \%$. Symptomatic response was observed in $96 \%$ of the patients. No grades 5, 4 and 3 acute and late toxicity was observed.

\section{CONCLUSION}

Continuous course reirradiation in patients with LRR in various carcinoma is feasible with acceptable toxicity. With current encouraging rates of local control and overall survival, this option should be discussed with patients who have few alternative therapeutic options.

\section{KEY WORDS}

Reirradiation, Recurrent, Radiation.

HOW TO CITE THIS ARTICLE: Katariya S, Verma M, Jain A, et al. An institutional study to evaluate the efficacy and toxicity of reirradiation using conventional radiotherapy for recurrent malignant disease. J. Evolution Med. Dent. Sci. 2019;8(03):174-176, DOI: $10.14260 /$ jemds/2019/38

\section{BACKGROUND}

So many times, in long term survivors of various cancer patients' recurrence may arise in previously radiated disease area. ${ }^{1}$ In such circumstances treatment of this recurrent disease is done traditionally by means other than radiotherapy that is chemotherapy and or surgery.

'Financial or Other Competing Interest': None.

Submission 19-12-2018, Peer Review 12-01-2019,

Acceptance 14-01-2019, Published 21-01-2019.

Corresponding Author:

Dr. Arpit Jain,

$3^{\text {rd }}$ Year Postgraduate Student,

Department of Radiation Oncology,

Government Cancer Hospital, MGMMC,

Indore, Madhya Pradesh, India.

E-mail: arpitjain8390@gmail.com

DOI: $10.14260 /$ jemds $/ 2019 / 38$

This approach is based on the belief that the tissue in the irradiated area will not tolerate a second course of Radiation therapy. In some cases, surgery is not feasible due to medical reasons while a few such patients adamantly refused to undergo any surgery while chemotherapy also does not give any result accept causing severe toxicity. Reirradiation with or without the addition of chemotherapy may hold promise for long-term survival for appropriately selected patients. ${ }^{2}$

\section{Purpose}

To evaluate the Efficacy and Toxicity of Reirradiation using conventional Radiotherapy for Recurrent Malignant Disease.

\section{MATERIALS AND METHODS Study Design \\ Prospective Observational Study.}


In this study, we present our results of reirradiation alone or reirradiation with concurrent chemotherapy of such patients. Out of a total twenty-six evaluable patients 10 patients were of malignancy in pelvis of which 9 were the patients of squamous cell carcinoma of uterine cervix and one patient of Ca Urinary bladder, with a median age of 57 year. 5 patients were of Brain Tumour, with a median age of 21 years, with histopathology of Medulloblastoma, Hemangiopericytoma and Astrocytoma Grade II (2 patients). One patient was diagnosed Brainstem glioma, only on CT scan findings. The remaining 11 patients were of Head and Neck squamous cell carcinoma including $\mathrm{Ca}$ tongue, Ca buccal mucosa, Ca larynx and Ca alveolus, with a median age of 55 years. A minimum disease-free interval after the initial radiotherapy treatment was considered to be of 1 year. The study was conducted during November 2014 to December 2017. Patients with Karnofsky Performance Status of 90-100 was included. Toxicity was evaluated according to Common Terminology Criteria for Adverse Events (CTCAE)

\section{RESULTS}

Out of 9 cancer cervix patients, four patients were treated with concomitant weekly CT with cisplatin and conventional RT (50 Gy in 25 fractions). All the four patients tolerated the treatment well and three responded very well with complete disappearance of the symptoms and visible growth. In remaining five patients four were treated by external RT only and in one patient intracavitary RT was also given and all of them responded very well with complete disappearance of disease.

One patient who presented with recurrence after 14 years of prior treatment, developed grade II skin reaction but after completion of treatment she was relieved of her pressure symptoms in pelvis with complete disappearance of visible growth. One patient treated with CTRT could tolerate the treatment well, but she could get only symptomatic relief from pain and bleeding, while the residual disease was persisting even after completion of re-radiation.

Patient with Ca urinary bladder had also responded very well to 200 cGy in 23 fractions of re-radiation along with 20 mg inj. Cisplatin D1 to D5 with complete disappearance of her symptoms and visible growth in USG.

Out of five different Brain Tumour patients, two patients were treated by conventional RT along with Tab
Temozolamide $100 \mathrm{mg}$ daily and they tolerated the treatment very well with marked symptomatic improvement. At the time of recurrence, the patient with Medulloblastoma had symptoms like disability in balancing, deafness right ear, sensational loss left half of the body with right facial palsy. All the symptoms disappear after completion of treatment. Similarly, at the time of recurrence, second patient (Astrocytoma Grade II) had symptoms like convulsion, weakness right half of the body, impaired memory and difficulty in writing. After completion of treatment there was about $90 \%$ improvement in the right hemiparesis, patient is started walking without support and also, he started writing. Two patients also have shown marked symptomatic relief and the patient with Hemangiopericytoma recovered completely, who was subjected to surgery also. While the second patient with Astrocytoma had some disability after the treatment. This patient was having the residual disease even after the treatment. One patient of 6-year age with brainstem glioma has also start recognising her mother father up to $40 \mathrm{~Gy}$ of radiation, but deteriorated After $50 \mathrm{~Gy}$ of external RT.

Out of 11 oral cavity cancer patients, two patients with Buccal mucosa disease and Retromolar area disease responded to the treatment (Concomitant CTRT) very well with complete disappearance of the growth and symptoms. While one patient with disease of Tongue had definitely shown marked regression of the growth with marked symptomatic relief in pain and bleeding but the disease was persisting even after completion of the treatment. Another Ca Tongue patient was cured of his disease with surgery \& RT. Similarly, in one patient with Ca Larynx, marked growth regression was observed but there was small residual disease after re-radiation. While another Ca Larynx patient was completely cured by node dissection and RT. Similarly, the patient with Angle of mouth disease was also having a small residual disease after completion of the reirradiation and refused surgery after reirradiation, which could have proved curative finally. One patient with Ca alveolus had residual diseases even after reirradiation, while another one had shown complete response with disappearance of disease. Patient with secondaries in neck with unknown primary disease had also tolerated and responded very well to Reirradiation.

\section{Characteristics}

\begin{tabular}{|c|c|c|c|}
\hline Patient characteristics & Pelvic tumors & Brain tumors & Head \& Neck Ca \\
\hline Total no. of Cases & 10 & 5 & 11 \\
\hline Male: female & $1: 9$ & $4: 1$ & $7: 4$ \\
\hline $\begin{array}{l}\text { Interval from previous } \\
\text { RT (mean, in months) }\end{array}$ & 62 & 28 & 22 \\
\hline KPS (PS) & $90-100$ & $90-100$ & $90-100$ \\
\hline $\begin{array}{l}\text { Treatment given } \\
\text { CTRT } \\
\text { RT alone }\end{array}$ & $\begin{array}{l}5 \\
5\end{array}$ & $\begin{array}{l}2 \\
3\end{array}$ & $\begin{array}{l}8 \\
3\end{array}$ \\
\hline Response & $\begin{array}{l}9 \\
1\end{array}$ & $\begin{array}{l}3 \\
2\end{array}$ & $\begin{array}{l}7 \\
4\end{array}$ \\
\hline $\begin{array}{l}\text { Complication } \\
\text { Acute (grade } 3 \& 4 \text { ) }\end{array}$ & 0 & $\begin{array}{c}\text { Large bowel obstruction (1 } \\
\text { Pt.) }\end{array}$ & $\begin{array}{l}\text { Mandibular Osteomyelitis } \\
\text { (1 Pt.) }\end{array}$ \\
\hline
\end{tabular}


No complication was observed in 24 out of these 26 patients. Only one patient of Ca cervix and one patient of buccal mucosa disease developed some difficulties later on, which could be attributed to the Reirradiation. In the patient of Ca cervix who had a recurrent disease after 14 years of prior treatment and retreated by radiotherapy alone, although she was relieved of her pressure symptoms of pelvis but after 8 months of the treatment, she developed large bowel obstruction. But this large bowel obstruction was already there prior to the second course of reirradiation due to parametrial Fibrosis. The patient refused to undergo colostomy and suffered repeated episodes of constipation and loose stools. Ultimately after 8 months of second course of radiotherapy she agreed for colostomy but died during the postoperative period with raised blood urea, anaemia and low general health.

The patient of Ca buccal mucosa who was re-radiated after 8 years of initial radiotherapy developed mandibular osteomyelitis about 11 months after the second course of radiotherapy and was subjected to partial mandibulectomy, excising only the affected part of mandible. Postoperative healing was uneventful, and the patient is disease and symptoms free since last one and half year except mild persisting oedema over the lateral part of lower lip.

\section{DISCUSSION}

Treatment of recurrent malignant disease is a difficult and complicated subject. In patients where trials of treatment with chemotherapy and/or surgery cannot be considered, reirradiation may be considered as a curative or a better palliative treatment option ${ }^{3}$. In a study of Re-radiation on pig skin and subcutaneous tissue, R.H. Sigmund et al found that the average reaction to re-radiation was significantly less than those for the comparable areas of skin that had not received radiation before. Similarly, Henry et al in his study on mouse tail confirms that after a 6 to 10 weeks period of previous radiation dose up to the tolerance level of mouse tail could tolerate an additional irradiation of $90 \%$ of previous dose. In a study undertaken by Wang CC in Massachusetts General Hospital Cancer Centre United States on 20 recurrent laryngeal carcinoma patients again it was concluded that the radiation tolerance of the laryngeal structure is much higher than it was commonly believed and reirradiation is possible and proved as a useful alternative to the surgery and therefore laryngectomy is reserved for failures. ${ }^{4}$ One study conducted in the department of radiation oncology of DDHCC (Dr Daniel Den Hoed Cancer Centre) of Rotterdam in the Netherlands and reported in 1990 also concluded that a median survival time is improve with re-radiation which is further batter if brachytherapy is also used. From this small study we also confirm that the radiation tolerance to reirradiation is much higher than we commonly believe for the head and neck areas and reirradiation has proved to be very important alternative treatment in recurrent head and neck cancers. Similarly, pelvic tissue and brain tissue have also tolerated the reirradiation as well with sufficiently good tumour response and symptomatic relief in recurrent brain tumour and complete tumour regression in recurrent cervical cancer.

\section{CONCLUSION}

Reirradiation has proved beneficial in recurrent head and neck cancers and this has been established by a number of workers in different institutions worldwide. This requires special observation to decide whether any such modality is worthwhile to anticipate a meaningful improvement in disease status and quality of life. ${ }^{5}$ Definitely in some cases it causes the disease while in some it is proved as a best palliative treatment without significant complications. Apart from the reirradiation of recurrent head and neck diseases, (Established in number of Institutions worldwide) further study is required to understand the role of reirradiation in recurrent cancers of other sides of the body and its complications particularly in Cancer Cervix and Brain Tumour as we tried in this study. Brain tissue could also tolerate Reirradiation as recurrent Brain Tumour has shown a considerably good response to re-radiation and improves the quality of life. In the end we would like to give emphasis on the fact that reirradiation preserves the organ and functions along with the better cosmesis. Therefore, we should give a thought to function preservation also, before deciding a definitive treatment for a case with recurrent malignant disease.

\section{REFERENCES}

[1] Brockstein B, Haraf DJ, Rademaker AW, et al. Patterns of failure, prognostic factors and survival in locoregionally advanced head and neck cancer treated with concomitant chemoradiotherapy: a 9-year, 337 patient, multi-institutional experience. Ann Oncol 2004;15(8):1179-86.

[2] Kao J, Garofalo MC, Milano MT, et al. Reirradiation of recurrent and second primary head and neck malignancies: a comprehensive review. Cancer Treat Rev 2003;29(1):21-30.

[3] Hoebers F, Heemsbergen W, Moor S, et al. Reirradiation for head-and-neck cancer: delicate balance between effectiveness and toxicity. Int J Radiat Oncol Biol Phys 2011;81(3):e111-8.

[4] Wang TJC, Riaz N, Tam M, et al. Patterns of failure after salvage re-irradiation for recurrent head and neck cancer: implications for field design and consolidation therapy. J Radiation Oncol 2014;3(2):139-45.

[5] Curtis KK, Ross HJ, Garrett AL, et al. Outcomes of patients with loco-regionally recurrent or new primary squamous cell carcinomas of the head and neck treated with curative intent reirradiation at Mayo Clinic. Radiat Oncol 2016;11:55. 